\title{
Characterization of the Ear Canal Bacterial Flora Present in Hearing Aids (HA) Wearing Subjects
}

\author{
Marta Aleandri1, Cecilia Ambrosi1,2, Maria Stefania Lepanto', Antonietta Lucia Conte1, \\ Massimiliano Marazzato ${ }^{1}$, Catia Longhi' ${ }^{1}$, Mauro Nicoletti ${ }^{3}$, Carlo Zagaglia1, Paola Goldoni', \\ Francesco Bussu4 4 , Roberta Anzivino4 ${ }^{4}$, Roberto Gallus ${ }^{5}$, Maria Pina Battista ${ }^{4}$, Maria Pia Conte ${ }^{*}{ }^{(0)}$, \\ Anna Teresa Palamara ${ }^{6,7}$
}

\author{
${ }^{1}$ Department of Public Health and Infectious Diseases, "Sapienza” University of Rome, Rome, Italy \\ ${ }^{2}$ Dani Di Giò Foundation-Onlus, Rome, Italy \\ ${ }^{3}$ Department of Medical, Oral and Biotechnological Sciences, University “G. D’Annunzio”, Chieti, Italy \\ ${ }^{4}$ Institute of Otolaryngology, "Cattolica del Sacro Cuore” University Agostino Gemelli Polyclinic, Rome, Italy \\ ${ }^{5}$ Otolaryngology, Mater Olbia Hospital, Olbia, Italy \\ ${ }^{6}$ Department of Public Health and Infectious Diseases, “Sapienza” University of Rome, Laboratory Affiliated to Institute Pasteur \\ Italia, Cenci-Bolognetti Foundation, Rome, Italy \\ ${ }^{7}$ San Raffaele Pisana, IRCCS, Telematic University, Rome, Italy \\ Email: *mariapia.conte@uniromal.it
}

How to cite this paper: Aleandri, M., Ambrosi, C., Lepanto, M.S., Conte, A.L., Marazzato, M., Longhi, C., Nicoletti, M., Zagaglia, C., Goldoni, P., Bussu, F., Anzivino, R., Gallus, R., Battista, M.P., Conte, M.P. and Palamara, A.T. (2019) Characterization of the Ear Canal Bacterial Flora Present in Hearing Aids (HA) Wearing Subjects. Advances in Microbiology, 9, 616-628.

https://doi.org/10.4236/aim.2019.97038

Received: May 31, 2019

Accepted: July 21, 2019

Published: July 24, 2019

Copyright $\odot 2019$ by author(s) and Scientific Research Publishing Inc. This work is licensed under the Creative Commons Attribution International License (CC BY 4.0).

http://creativecommons.org/licenses/by/4.0/ CC) (7) Open Access

\begin{abstract}
The use of hearing aids (HA) is considered a predisposing factor for ear microbial infections. We undertook this study to compare the presence and nature of the microbial flora inhabiting of ears of HA and non-HA (nHA) users. Swab samples of the ears of HA and nHA users were collected from the Institute of Otolaryngology, "Cattolica del Sacro Cuore" University "Agostino Gemelli”, Rome, Italy. Swab samples were taken from the ear canal of 57 HA and $33 \mathrm{nHA}$ users. The components of the microbial flora present on each swab sample were identified and characterized at the level of species. A total of 41 different bacterial species were identified. A statistically significant prevalence of polymicrobial communities was found in ears presenting signs of inflammation $(2.5 \pm 1.7$ vs $2.1 \pm 1.3 ; \mathrm{P}=0.02)$ and in HA users $(2.3 \pm 1.2$ vs $1.7 \pm 1.0 ; \mathrm{P}=0.002$ ). Few putative pathogens were detected. Candida albicans spp. was not isolated in our study. A small number of swab samples presented no microbial growth. Bacterial species isolated from HA users with and without inflammation were assayed for the ability to form biofilm. Among gram-positive and gram-negative bacteria, $S$. aureus, CoNS, $P$. aeruginosa and $K$. pneumoniae were found to be strong biofilm producers. $S$. aureus and
\end{abstract}


$P$. aeruginosa, isolated only from the ears of $\mathrm{HA}$ and nHA users presenting signs of inflammation, were further analyzed for their antibiotic-resistance profile and characterized by the Multilocus Sequence Typing (MLST) assay. The highest rates of antibacterial resistance were in $S$. aureus to penicillin (75.5\%) and in $P$. aeruginosa, to amoxicillin-clavulanic acid, cefotaxime, ertapenem, tigecycline and trimethoprim-sulfamethoxazole (100\%). Moreover, three $S$. aureus strains (37.5\%) were methicillin-resistant (MRSA). Of the eight $S$. aureus isolates, we identified six sequence types (ST) indicating that $75 \%$ are likely independent clones. For what it concerned $P$. aeruginosa, six different STs were assigned. Interestingly, two out of the six strains presented newly identified ST values. This study sheds new light on the combined effect of the presence of HA devices and signs of external ear inflammation on the composition of the ear bacterial flora. Our results reinforce the need to practice careful hygiene of HA devices to prevent serious ear canal infections.

\section{Keywords}

Ear Bacterial Flora, Hearing Aids, Acute Otitis Externa

\section{Introduction}

There are many causes of hearing loss [1]. Sensorineural presbycusis is the most common type of hearing loss, especially in elderly people, and hearing aids (HA) are the mainstay of treatment, that can improve patient quality of life. However, it is well known that the use of HAs could be a predisposing factor for the development of bacterial infections that may lead to acute otitis externa (AOE) [1]-[7], a disease with a high incidence in the community population (approximately $1 \%$ $10 \%$, with a peak in summer) [8]. Clinically, AOE consists of a microbial infection followed by severe inflammation of the external ear canal, often associated with ear pain, swelling, fluid accumulation, and rarely fever. Chronic, long-standing or recurrent middle ear infections often result in both conductive and sensorineural presbycusis. Bacteria and occasionally fungi (principally Staphylococcus aureus, Pseudomonas aeruginosa, and Candida albicans) have been associated with the development of AOE. Nevertheless, also a variety of non-pathogenic microorganisms or local dermatologic processes have been implicated in AOE development [9]. Among the auditory defenses, cerumen has been reported to contain substances that may counteract the action of microbial infections [10] [11].

It has been previously reported that carriage of HA devices modifies the ear microbiota, favoring the proliferation of both commensal and pathogenic bacteria, thus increasing the risk of AOE [3] [12] [13] [14]. Recently, AOE caused by highly antibiotic-resistant bacteria is an emerging concern for clinicians to eradicate these infections. Although there are several reports regarding the microorganisms involved in AOE, to date only a few studies are available on the characterization of the ear microbiological flora of healthy and patients with $\mathrm{AOE}$ 
with or without HA [2] [15].

This study is focused on the characterization of the microbial flora present in HA and non-HA (nHA) users with or without signs of inflammation. Moreover, biofilm production, allelic and antibiotic resistance profiles were also determined for some bacterial prototypes.

\section{Materials and Methods}

\subsection{Study Subjects}

Fifty-seven hearing-impaired patients (39 men and 18 women) wearing hearing aids (HA) and 33 normal subjects (15 men and 18 women), ranging from 7 to 79 years of age (mean 49.5 years), were enrolled in this study. Patients were informed about the purpose of the study and they gave a written informed consent. Ethical committee approval was obtained from the Ethical Committee of the University “Cattolica del Sacro Cuore”, Policlinico “A. Gemelli”, Rome, Italy (registration No. 20532/13). The otoscopic examination was carried out to detect signs of inflammation at the external auditory canal. Inflammation was defined mild when edema and occasionally clear secretions were present or moderate when edema, secretions, and debris of irritated skin were detected. Patients with active ear discharge, tympanic ear perforation, or that had taken any antimicrobial treatment in the preceding seven days, were excluded from this study. Ear swab samples of the left ears (taken without cleaning of any debris/exudates or cerumen) of subjects with or without HA and with or without signs of inflammation were collected from February 2016 to September 2017. Samples were collected and placed in tubes containing sterile Amies transport culture medium with charcoal (Sterile transport swab, Oxoid) and transported to the Microbiology Laboratory for microbial isolation and characterization. HAs were removed just before sampling. Samples were taken in spring to avoid seasonal colonization differences and to equalize the experimental conditions.

\subsection{Microbial Identifications}

Swabs were independently streaked onto a set of culture media plates, namely Blood agar, Chocolate agar supplemented or not with bacitracin, Mannitol Salt Agar, MacConkey 3 agar, Bile-esculin agar, Cetrimide, and Chromogenic Candida agar plates (all from Oxoid). Plates were incubated for $24-48 \mathrm{hrs}$ at 25 and $37^{\circ} \mathrm{C}$ in the presence or not of an atmosphere of $5 \% \mathrm{CO}_{2}$. Sabouraud Dextrose agar plates were incubated up to seven days. A load of bacteria on the swabs was estimated by immersing the swabs in sterile tubes containing $1.0 \mathrm{ml}$ of PBS for 1 $\mathrm{h}$ to release microorganisms. Then, a volume of $0.1 \mathrm{ml}$ of this solution was streaked on suitable culture plates and incubated at $37^{\circ} \mathrm{C}$ for $48 \mathrm{hrs}$. The bacterial load was estimated as low $\left(\leq 10^{3} \mathrm{CFU} \cdot \mathrm{ml}^{-1}\right)$, medium $\left(10^{3}-10^{4} \mathrm{CFU} \cdot \mathrm{ml}^{-1}\right)$ and heavy $\left(\geq 10^{4}-10^{5} \mathrm{CFU} \cdot \mathrm{ml}^{-1}\right)$. Isolates were identified at the level of species by conventional microbiological methods (API ${ }^{\circledR}$ System and Vitek-2 Bio-Mèrieux) or by $16 \mathrm{~S}$ rDNA gene sequencing. 


\subsection{S rDNA Gene Sequencing}

The 16S rDNA gene sequencing was used to identify bacterial species that could not be identified by conventional biochemical methods. Briefly, total DNA was extracted by suspending three to five colonies in TE buffer $(10 \mathrm{mM}$ Tris, $1 \mathrm{mM}$ EDTA, $\mathrm{pH}$ 7.8) and heating at $100^{\circ} \mathrm{C}$ for $20 \mathrm{~min}$. After centrifugation, supernatants were collected for PCR analysis. Universal primers

8 F (5'-AGAGTTTGATCCTGGCTCAG-3') and 1492R (5'-GGTTACCTTGTTACGACTT-3') were used to amplify the 16S rDNA gene [16]. Amplicons were visualized by $1 \%$ agarose gel electrophoresis to confirm the presence of the expected 1500-bp fragment. Amplicons were quantified and sequenced bi-directionally using the same primers described above. Alignment and comparison of the $16 \mathrm{~S}$ rDNA sequences were performed using the BLAST tool (https://pubmlst.org).

\subsection{Multilocus Sequence Typing}

Multilocus sequence typing (MLST) was used to determine genetic relatedness among of $S$. aureus and $P$. aeruginosa isolates. Species-specific primer pairs were used to amplify and sequence internal fragments of two sets of seven housekeeping genes, as previously reported for $\mathcal{S}$. aureus [17] and $P$. aeruginosa [18]. Total DNA extracts were prepared by using the GenElute Bacterial Genomic DNA system (Qiagen) according to the manufacturer's instructions. Amplification and sequencing were performed at the BMR Genomics, Padova, Italy by standard sequencing methods.

Sequence types (ST) were determined by comparing the obtained sequences with the specific databases hosted at https://pubmlst.org. Only perfect matches were considered to assign amplified fragments to specific alleles.

\subsection{Antimicrobial Susceptibility Tests}

Antibiotic-resistance profiles of the eight $S$. aureus and of the six $P$. aeruginosa isolates was determined according to the guidelines of the European Committee on Antimicrobial Susceptibility Testing (EUCAST, version 6.0). Tests were performed using the automated microbial system Vitek-2 (Bio-Mèrieux). Minimal inhibitory concentrations (MICs) were also determined.

\subsection{Biofilm Assay}

The more prevalent bacteria species isolated from HA users with and without inflammation were assayed for the ability to form biofilm as previously described [19] [20]. Escherichia coli strain 16 [19] and Staphylococcus epidermidis ATCC RP62A [20] were used as positive controls.

Biofilms were quantified by measuring the absorbance at $\lambda 595 \mathrm{~nm}\left(A_{595}\right)$ with a microplate reader (Tecan Sunrise, X-fluor). A cut-off value of optical density (ODc), defined as the OD average of non-inoculated medium and a three standards deviation, was established. Strains were defined non-biofilm producers 
when the OD values were OD $\leq$ ODc; weak biofilm producers when OD values were $\mathrm{ODc} \leq \mathrm{OD} \leq 2 \mathrm{ODc}$; moderate biofilm producers when $\mathrm{OD}$ values were $2 \mathrm{ODc} \leq \mathrm{OD} \leq 4 \mathrm{ODc}$ and strong biofilm producers when $\mathrm{OD}$ values were $\mathrm{OD} \geq$ $4 \mathrm{ODc}[20]$.

\subsection{Statistical Analysis}

The differences in the distribution of species and monomicrobial and polymicrobial communities among groups were calculated using the $\chi 2$ test (with Yates' continuity correction). For species richness, a bilateral Mann-Whitney U-test was used to compare groups. In both cases, a $P$ value $\leq 0.05$ was considered statistically significant.

\section{Results}

\subsection{Ears Sampling}

A total of 90 subjects were enrolled in this study. Swab samples were taken from left ears of $57 \mathrm{HA}$ and $33 \mathrm{nHA}$ users. Prior to sampling, the otoscope examination was used to detect signs of external auditory canal inflammation. Signs of mild/moderate inflammation were found in 22 subjects ( 10 out the $57 \mathrm{HA}$ and 12 out the 33 nHA users).

\subsection{Microbiological Characterization of Ear Samples}

A variety of microorganisms were recovered from swab cultures of the 90 subjects and a total of 41 different bacterial species (29/41 Gram + and 12/41 Gram -) were identified. Bacteria considered part of the ear normal flora were the most common isolates. Among these, the majority were Coagulase-Negative Staphylococci (CoNS in Table 1), followed by Corynebacterium spp., Bacillus spp., and other microorganisms (Table 1). On the other hand, few putative pathogenic microorganisms, namely $S$. aureus, $S$. agalactiae, $E$. coli, E. cloacae, $K$. pneumoniae and $P$. aeruginosa were isolated from 43 out of the 90 cultured swabs (47.7\%). Putative pathogenic $S$. aureus and $P$. aeruginosa strains were isolated only from HA and nHA users presenting signs of inflammation. No strains of Candida spp. were found. No microbial growth was observed with a statistically significant prevalence among nHA compared to HA users (6 out of 33 vs 1 out of 57, respectively; $P=0.032$ ).

Moreover, the statistically significant prevalence of the bacterial species in with respect to the different groups of patients was evaluated. In this analysis, we found that the predominant microorganism recovered was CoNS found in the great majority of samples $(91.1 \%, 82 / 90)$, independently from the presence of signs of inflammation (respectively, 22/22 patients with inflammation vs 60/68 patients without signs of inflammation, $P<0.001$ ) (Table 1). Moreover, Corynebacterium spp. and Micrococcus spp. were statistically prevalent in ears presenting signs of inflammation (respectively, $4 / 22$ vs $1 / 68, P<0.012$ and $4 / 22 \mathrm{vs}$ $2 / 68 ; P<0.03$ ). On the other hand, Bacillus spp. was statistically prevalent among 
Table 1. Microbiological analysis of ear bacterial flora.

\begin{tabular}{|c|c|c|c|c|c|c|c|c|}
\hline \multirow{2}{*}{$\begin{array}{l}\text { Microorganisms } \\
\text { Gram-positive }\end{array}$} & \multicolumn{4}{|c|}{ HA } & \multicolumn{4}{|c|}{ nHA } \\
\hline & \multicolumn{2}{|c|}{$\begin{array}{c}\text { HA users } \\
\text { presenting signs of } \\
\text { inflammation (n. 10) }\end{array}$} & \multicolumn{2}{|c|}{$\begin{array}{l}\text { HA users without } \\
\text { any signs of } \\
\text { inflammation (n. 47) }\end{array}$} & \multicolumn{2}{|c|}{$\begin{array}{c}\text { nHA users } \\
\text { presenting signs of } \\
\text { inflammation (n. 12) }\end{array}$} & \multicolumn{2}{|c|}{$\begin{array}{l}\text { nHA users without } \\
\text { any signs of } \\
\text { inflammation (n. 21) }\end{array}$} \\
\hline & & & & & & & & \\
\hline Corynebacterium spp. & $2 / 10$ & $20 \%$ & 1 & $2.1 \%$ & 2 & $16.7 \%$ & - & - \\
\hline Brevibacterium sanguinis & - & - & - & - & 1 & $8.3 \%$ & - & - \\
\hline Kocuria varians/rosea & - & - & - & - & 1 & $8.3 \%$ & - & - \\
\hline Micrococcus spp. & $2 / 10$ & $20 \%$ & - & - & 2 & $16.7 \%$ & 2 & $9.5 \%$ \\
\hline Bacillus spp. & $1 / 10$ & $10 \%$ & 19 & $40.4 \%$ & - & - & - & - \\
\hline Gemella haemolysans & - & - & 1 & $2.1 \%$ & - & - & - & - \\
\hline Staphylococcus aureus & $4 / 10$ & $40 \%$ & & & 4 & $33.3 \%$ & - & \\
\hline CoNS & $10 / 10$ & $100 \%$ & 42 & $89.4 \%$ & 12 & $100 \%$ & 18 & $85.7 \%$ \\
\hline Streptococccus agalactiae & - & - & - & - & 1 & $8.3 \%$ & - & - \\
\hline Enterococcus faecalis & - & - & - & - & 1 & $8.3 \%$ & - & - \\
\hline Aerococcus viridans & $1 / 10$ & $10 \%$ & - & - & - & - & - & - \\
\hline \multicolumn{9}{|l|}{ Gram-negative } \\
\hline Citrobacter freundii & $2 / 10$ & $20 \%$ & - & - & - & - & - & - \\
\hline Escherichia coli & $1 / 10$ & $10 \%$ & 6 & $12.8 \%$ & - & - & - & - \\
\hline Enterobacter cloacae & $4 / 10$ & $40 \%$ & 9 & $19.1 \%$ & - & - & - & - \\
\hline Enterobacter ludwigii. & $1 / 10$ & $10 \%$ & - & - & - & - & - & - \\
\hline Klebsiella pneumoniae & $1 / 10$ & $10 \%$ & 6 & $12.7 \%$ & - & - & - & - \\
\hline Klebsiella variicola. & $1 / 10$ & $10 \%$ & - & - & - & - & - & - \\
\hline Pantoea agglomerans & - & - & 1 & $2.1 \%$ & - & & - & - \\
\hline Pseudomonas aeruginosa & $1 / 10$ & $10 \%$ & - & - & 5 & $41.7 \%$ & - & - \\
\hline Pseudomonas luteola & - & - & 2 & $4.3 \%$ & - & - & - & - \\
\hline Achromobacter spp. & - & - & 1 & $2.1 \%$ & - & - & - & - \\
\hline Ralstonia pickettii & $1 / 10$ & $10 \%$ & - & - & - & & - & - \\
\hline
\end{tabular}

Microbiological analysis of bacterial flora colonizing the ear canal of HA and nHA users presenting or not inflammation. Isolates were identified at the level of species by conventional microbiological methods and $16 \mathrm{~S}$ rDNA sequencing. The number of bacterial species and the percentage of isolates are indicated. Corynebacterium spp. were $C$. afermentas, $C$. aurimucosum, $C$. pseudodiftericum, and Corynebacterium of unknown species. Bacillus spp. were B. subtilis, B. amyloliquefaciens, B. licheniformis, B. mojavensis, B. pseudomycoides, B. pumilus, B. simplex and Bacillus of unknown species. CoNS were $S$. auricularis, S. capitis, S. epidermidis, S. haemolyticus, S. hominis, S. sciuri, S. warneri, S. warneri/pasteuri and S. xylosus.

HA users $(20 / 57$ vs $0 / 33 ; P<0.019)$ (Table 1$)$. For what it regards putative pathogenic strains, $E$. cloacae was isolated only among HA users (13/57 vs $0 / 33 ; P$ $=0.02$ ) while $P$. aeruginosa (six isolates) and $S$. aureus (eight isolates) were exclusively recovered from ears presenting signs of inflammation (respectively, $6 / 22$ vs $0 / 68 ; P<0.0001$ and $8 / 22$ vs $0 / 68 ; P<0.0001$ ).

Polybacterial flora, mainly composed of commensals, was recovered with a 
statistically significant prevalence among nHA respect to HA users (27/33 vs $40 / 57, P<0.049)$. A deeper evaluation of the complexity of these bacterial communities was conducted by determining species richness and the average number of bacterial species recovered per swab sample. To this end, the number of bacterial species recovered from HA and nHA users, with or without signs of inflammation, were compared and polymicrobial communities were statistically prevalent in subjects with signs of inflammation respect to subject without inflammations $(2.5 \pm 1.7$ vs $2.1 \pm 1.3 ; P=0.02)$. Consequently, polymicrobial communities were confirmed to be statistically prevalent in $\mathrm{nHA}$ in with respect to HA users $(2.3 \pm 1.2$ vs $1.7 \pm 1.0 ; P=0.002)$. Taken together these results indicate that the presence of HA as well as of inflammation are conditions that may favor the formation of polybacterial communities within the external ear canal.

\subsection{Antibiotic-Resistance Profiles of $S$, aureus and $P$. aeruginosa Strains Isolated from HA and nHA Users Presenting Signs of Inflammation}

Since $S$. aureus and $P$. aeruginosa were recovered only from HA and of nHA users presenting signs of inflammation (Table 1), we decided to better characterize these strains. To this end, we first determined their antibiotic-resistance profiles. The antibiotics used (Oxoid) are reported on the legends of Table 2 and Table 3. All strains of $S$. aureus were sensitive (S) to a variety of antibiotics, namely linezolid, daptomycin, vancomycin, tigecycline, fusidic acid, rifampicin and trimethoprim-sulfamethoxazole. Almost all strains were resistant (R) to penicillin $G$, three strains were methicillin-resistant (MRSA), and presented different antibiotic resistance profiles (Table 2). For what it concerned $P$. aeruginosa, the strains showed very similar antibiotic-resistance profiles (they were $100 \%$ resistant to amoxicillin-clavulanic acid, cefotaxime, ertapenem, tigecycline and trimethoprim-sulfamethoxazole) (Table 3).

\subsection{Allelic Variation in $S$, aureus and $P$. aeruginosa}

To determine genetic relatedness among the $S$. aureus and $P$. aeruginosa strains, an MLST typing assay was performed, as described in Materials and Methods. The specific housekeeping genes and the primers used to amplify internal DNA fragments of $S$. aureus and $P$. aeruginosa were previously described [17] [18] (Table 4 and Table 5). The PCR amplicons obtained were sequenced and sequences were assigned as distinct alleles by comparison with available specific MLST database. Subsequently, the sequence type (ST) values, for each isolate, were determined basing on its allelic profile. Table 4 and Table 5 show the different allelic profiles for each $S$. aureus and $P$. aeruginosa isolates. Of the eight $S$. aureus isolates, we identified six STs indicating that $75 \%$ are likely independent clones. In fact, two strains were assigned to ST8 and two to ST15 (Table 4) indicating that they represent genetically related clones. For what it concerned $P$. aeruginosa, the results obtained clearly indicated that they were unrelated to each other (six different ST values were assigned). Interestingly, two out of the 
Table 2. Antibiotic resistance profiles of $S$. aureus strains.

\begin{tabular}{|c|c|c|c|c|c|c|c|c|c|}
\hline$S$. aureus isolates from: & Strains & penG & met & gen & \multicolumn{2}{|c|}{$\operatorname{lv} x$} & ery & cli & lzd \\
\hline \multirow{4}{*}{$\begin{array}{l}\text { nHA users presenting } \\
\text { signs of inflammation }\end{array}$} & ORL 001 & $R(\geq 0.5)$ & $S(\leq 0.25)$ & $S(\leq 0.5$ & & $.12)$ & $S(0.5)$ & $S(0.25)$ & $S(2)$ \\
\hline & ORL 009 & $\mathrm{R}(\geq 0.5)$ & $\mathrm{R}(\geq 4)$ & $S(\leq 0.5)$ & \multicolumn{2}{|c|}{$\mathrm{S}(\leq 0.12)$} & $\mathrm{R}(\geq 0.8)$ & $\mathrm{R}(0.5)$ & $S(2)$ \\
\hline & ORL 011 & $S(\leq 0.03)$ & $S(\leq 0.25)$ & $S(\leq 0.5)$ & \multicolumn{2}{|c|}{$S(0.25)$} & $S(1)$ & $S(0.25)$ & $S(2)$ \\
\hline & ORL 024 & $\mathrm{R}(\geq 0.5)$ & $\mathrm{R}(\geq 4)$ & $S(\leq 0.5)$ & \multicolumn{2}{|c|}{$S(0.25)$} & $\mathrm{R}(\geq 0.8)$ & $S(0.25)$ & $S(2)$ \\
\hline \multirow{4}{*}{$\begin{array}{l}\text { HA users presenting } \\
\text { signs of inflammation }\end{array}$} & ORL 028 & $\mathrm{R}(\geq 0.5)$ & $\mathrm{R}(\geq 4)$ & $\mathrm{R}(\geq 16)$ & \multicolumn{2}{|c|}{$S(\leq 0.12)$} & $S(1)$ & $S(0.25)$ & $S(2)$ \\
\hline & ORL 094 & $\mathrm{R}(\geq 0.5)$ & $S(\leq 0.25)$ & $S(\leq 0.5)$ & \multicolumn{2}{|c|}{$S(\leq 0.12)$} & $S(1)$ & $S(0.25)$ & $S(2)$ \\
\hline & ORL 099 & $\mathrm{R}(\geq 0.5)$ & $S(0.5)$ & $S(\leq 0.5)$ & \multicolumn{2}{|c|}{$S(0.25)$} & $S(1)$ & $S(0.25)$ & S (2) \\
\hline & ORL OC & $\mathrm{R}(0.25)$ & $S(\leq 0.25)$ & $S(\leq 0.5)$ & \multicolumn{2}{|c|}{$S(\leq 0.12)$} & $S(1)$ & $S(0.25)$ & $S(2)$ \\
\hline$S$. aureus isolates from: & Strains & dap & tec & van & tet & $\operatorname{tgc}$ & fus & rif & sxt \\
\hline \multirow{4}{*}{$\begin{array}{l}\text { nHA users presenting } \\
\text { signs of inflammation }\end{array}$} & ORL 001 & $S(0.25)$ & $S(\leq 0.5)$ & $S(\leq 0.5)$ & $\mathrm{S}(\leq 1)$ & $S(\leq 0.12)$ & $S(\leq 0.5)$ & $S(\leq 0.03)$ & $S(\leq 10)$ \\
\hline & ORL 009 & $S(0.25)$ & $S(\leq 0.5)$ & $S(1)$ & $\mathrm{R}(\geq 16)$ & $\mathrm{S}(\leq 0.12)$ & $S(\leq 0.5)$ & $S(\leq 0.03)$ & $S(\leq 10)$ \\
\hline & ORL 011 & $S(0.25)$ & $S(\leq 0.5)$ & $S(1)$ & $\mathrm{S}(\leq 1)$ & $S(\leq 0.12)$ & $S(\leq 0.5)$ & $S(\leq 0.03)$ & $S(\leq 10)$ \\
\hline & ORL 024 & $S(0.25)$ & $S(\leq 0.5)$ & $S(1)$ & $S(\leq 1)$ & $S(\leq 0.12)$ & $S(\leq 0.5)$ & $S(\leq 0.03)$ & $S(\leq 10)$ \\
\hline & ORL 028 & $S(0.25)$ & $S(\leq 0.5)$ & $S(1)$ & $S(\leq 1)$ & $S(\leq 0.12)$ & $S(\leq 0.5)$ & $S(\leq 0.03)$ & $S(\leq 10)$ \\
\hline HA users presenting & ORL 094 & $\mathrm{~S}(0.25)$ & $S(\leq 0.5)$ & $S(1)$ & $\mathrm{R}(\geq 16)$ & $S(\leq 0.12)$ & $S(\leq 0.5)$ & $S(\leq 0.03)$ & $S(\leq 10)$ \\
\hline signs of inflammation & ORL 099 & $S(0.5)$ & $S(\leq 0.5)$ & $S(1)$ & $S(\leq 1)$ & $\mathrm{S}(\leq 0.12)$ & $S(\leq 0.5)$ & $S(\leq 0.03)$ & $S(\leq 10)$ \\
\hline & ORL OC & $S(0.25)$ & $\mathrm{R}(\geq 0.5)$ & $S(\leq 32)$ & $\mathrm{R}(\geq 16)$ & $\mathrm{S}(\leq 0.12)$ & $S(\leq 0.5)$ & $S(\leq 0.03)$ & $S(\leq 10)$ \\
\hline
\end{tabular}

Antibiotic resistance profiles of the eight $S$. aureus strains isolated from the ears of HA and nHA users presenting signs of inflammation. Isolates were defined antibiotic susceptible (S) or resistant (R). Antibiotics used were: PenG: benzylpenicillin; met: methicillin; gen: gentamicin; lvx: levofloxacin; ery: erythromycin; cli: clindamycin; lzd: linezolid; dap: daptomycin; tec: teicoplanin; van: vancomycin; tet: tetracyclin; tgc: tigecycline; fus: fusidic; acid; rif: rifampicin; stx: trimethoprim-sulfamethoxazole.

Table 3. Antibiotic resistance profiles of $P$. aeruginosa strains.

\begin{tabular}{|c|c|c|c|c|c|c|c|c|}
\hline P. aeruginosa isolates from: & Strains & amc & tzp & ctx & caz & cef & etp & imp \\
\hline \multirow{5}{*}{$\begin{array}{l}\text { nHA users presenting } \\
\text { signs of inflammation }\end{array}$} & ORL 001 & $\mathrm{R}(\geq 32)$ & S (8) & $\mathrm{R}(16)$ & S (2) & $S(0.5)$ & $\mathrm{R}(\geq 8)$ & S (2) \\
\hline & ORL 006 & $\mathrm{R}(\geq 32)$ & $\mathrm{S}(\leq 4)$ & $\mathrm{R}(16)$ & $S(2)$ & $S(8)$ & $\mathrm{R}(\geq 8)$ & $S(2)$ \\
\hline & ORL 011 & $\mathrm{R}(\geq 32)$ & S (8) & $\mathrm{R}(16)$ & S (2) & $\mathrm{S}(\leq 1)$ & $\mathrm{R}(\geq 8)$ & $S(1)$ \\
\hline & ORL 016 & $\mathrm{R}(\geq 32)$ & $S(8)$ & $\mathrm{R}(\geq 64)$ & S (4) & $S(2)$ & $\mathrm{R}(\geq 8)$ & $S(0.5)$ \\
\hline & ORL 025 & $\mathrm{R}(\geq 32)$ & $S(8)$ & $\mathrm{R}(32)$ & S (4) & $S(2)$ & $\mathrm{R}(4)$ & $S(1)$ \\
\hline $\begin{array}{l}\text { HA users presenting } \\
\text { signs of inflammation }\end{array}$ & ORL 074 & $\mathrm{R}(\geq 32)$ & $\mathrm{S}(\leq 4)$ & $\mathrm{R}(16)$ & $S(2)$ & $\mathrm{S}(\leq 1)$ & $\mathrm{R}(\geq 0.8)$ & $S(2)$ \\
\hline$P$. aeruginosa isolates from: & Strains & mem & amk & gen & cip & $\operatorname{tgc}$ & cst & sxt \\
\hline \multirow{5}{*}{$\begin{array}{l}\text { nHA users presenting } \\
\text { signs of inflammation }\end{array}$} & ORL 001 & $S(1)$ & $\mathrm{S}(\leq 2)$ & $\mathrm{S}(\leq 1)$ & $S(\leq 0.25)$ & $\mathrm{R}(\geq 8)$ & $\mathrm{S}(\leq 0.5)$ & $\mathrm{R}(80)$ \\
\hline & ORL 006 & $S(\leq 0.25)$ & S (4) & S (4) & $S(\leq 0.25)$ & $\mathrm{R}(\geq 8)$ & $S(\leq 0.5)$ & $\mathrm{R}(160)$ \\
\hline & ORL 011 & $\mathrm{~S}(\leq 0.25)$ & $\mathrm{S}(\leq 2)$ & $\mathrm{S}(\leq 1)$ & $S(\leq 0.25)$ & $\mathrm{R}(\geq 8)$ & $S(\leq 0.5)$ & $\mathrm{R}(80)$ \\
\hline & ORL 016 & $S(\leq 0.25)$ & $S(\leq 2)$ & $\mathrm{S}(\leq 1)$ & $S(\leq 0.25)$ & $\mathrm{R}(\geq 8)$ & $S(\leq 0.5)$ & $\mathrm{R}(160)$ \\
\hline & ORL 025 & $S(\leq 0.25)$ & $S(\leq 2)$ & $S(\leq 1)$ & $S(\leq 0.25)$ & $\mathrm{R}(\geq 8)$ & $S(\leq 0.5)$ & $\mathrm{R}(\geq 320)$ \\
\hline $\begin{array}{l}\text { HA users presenting } \\
\text { signs of inflammation }\end{array}$ & ORL 074 & $S(\leq 0.25)$ & $S(\leq 2)$ & $S(\leq 1)$ & $S(\leq 0.25)$ & $\mathrm{R}(\geq 8)$ & $S(\leq 0.5)$ & $\mathrm{R}(\geq 320)$ \\
\hline
\end{tabular}

Antibiotic resistance profiles of the six $P$. aeruginosa strains isolated from the ears of HA and nHA users presenting signs of inflammation. Isolates were defined antibiotic susceptible (S) or resistant (R). Antibiotics used were: amc: amoxicillin-clavulanic acid; tzp: piperacillin-tazobactam; ctx: cefotaxime; caz: ceftazimide; cef: cefepime; etp: ertapenem; imp: imipenem; mem: meropenem; amk: amikacin; gen: gentamicin; cip: ciprofloxacin; tgc: tigecycline; cst: colistin; sxt: trimethoprim-sulfamethoxazole. 
Table 4. Multi locus sequence typing analysis of $S$. aureus isolates.

\begin{tabular}{|c|c|c|c|c|c|c|c|c|c|}
\hline \multirow{2}{*}{ Strains } & \multicolumn{7}{|c|}{ Alleles } & \multirow{2}{*}{ ST } & \multirow{2}{*}{ HA } \\
\hline & $\operatorname{arcC}$ & aroE & $g l p F$ & $g m k$ & Pta & $t p i$ & $y q i L$ & & \\
\hline ORL001 & 7 & 6 & 1 & 70 & 8 & 8 & 6 & 737 & - \\
\hline ORL009 & 1 & 1 & 1 & 1 & 1 & 1 & 1 & 1 & - \\
\hline ORL011 & 5 & 4 & 1 & 4 & 4 & 6 & 3 & 7 & - \\
\hline ORL024 & 3 & 3 & 1 & 1 & 4 & 4 & 3 & 8 & - \\
\hline ORL028 & 3 & 3 & 1 & 1 & 4 & 4 & 3 & 8 & + \\
\hline ORL094 & 2 & 2 & 2 & 2 & 15 & 3 & 2 & 37 & + \\
\hline ORL099 & 13 & 13 & 1 & 1 & 12 & 11 & 13 & 15 & + \\
\hline ORLOC & 13 & 13 & 1 & 1 & 12 & 11 & 13 & 15 & + \\
\hline
\end{tabular}

Allelic variations of $S$. aureus strains isolated from the ears. Multilocus Sequence Typing (MLST) assays were conducted. ST: sequence type; HA-: strains isolated from nHA users; HA+: strains isolated from HA users.

Table 5. Multi locus sequence typing analysis of $P$. aeruginosa isolates.

\begin{tabular}{ccccccccccc}
\hline \multirow{2}{*}{ Strains } & \multicolumn{7}{c}{ Alleles } & ST & HA \\
\cline { 2 - 8 } & acs $A$ & aroE & gua $A$ & mutL & nuoD & ppsA & trpE & & \\
\hline ORL001 & 67 & 5 & 12 & 3 & 14 & 4 & 7 & new & + \\
ORL006 & 6 & 5 & 6 & 7 & 4 & 6 & 7 & 27 & - \\
ORL011 & 13 & 75 & 5 & 5 & 12 & 7 & 15 & 481 & - \\
ORL016 & 9 & 87 & 5 & 5 & 1 & 6 & 47 & new & - \\
ORL025 & 27 & 91 & 5 & 3 & 1 & 6 & 64 & 522 & - \\
ORL074 & 6 & 5 & 6 & 3 & 74 & 13 & 7 & 672 & - \\
\hline
\end{tabular}

Allelic variations of $P$. aeruginosa strains isolated from the ears. Multilocus Sequence Typing (MLST) assays were conducted. ST: sequence type; HA-: strains isolated from nHA users; HA+: strains isolated from HA users.

six strains presented newly identified ST values (Table 5). These results indicated that the $P$. aeruginosa isolates are likely independent clones, thus confirming previous reports indicating the high genetic variability of this facultative pathogen [18].

\subsection{Biofilm Production}

The ability to produce biofilm was evaluated, as described in Materials and Methods, for all different bacterial species isolated from HA users, with or without signs of inflammation. In general, biofilm production was found to be statistically not correlated to the presence of signs of inflammation. Gram-positive bacteria, such as $S$. aureus and CoNS, were found to be strong biofilm producers. Among Gram-negative putative bacterial pathogens, all strains of $P$. aeruginosa, $K$. pneumoniae, were strong biofilm producers, while E. coli and E. cloacae appeared weak/moderate biofilm producers (data not shown). Taken together our 
results indicated that biofilm production appears to be a quite diffuse property among bacterial species colonizing the ear canal of HA users, independently from inflammation. This may contribute to the development of infection to the external ear canal and likely to the progression to acute otitis externa (AOE).

\section{Discussions}

One relevant characteristic of the external auditory canal is its unique cul-de-sac anatomical structure that readily traps moisture thereby favoring colonization and growth of a wide range of microorganisms (either commensal or pathogens). Moisture retention, caused by the presence of hearing aids (HA), may favor occlusion of the ear canal and thus bacterial colonization also of the HA device by biofilm-producing microorganisms. Thus, HA carriage, especially if it is not followed by daily efficient cleaning and disinfection of the HA device, is considered a risk factor for the development of external canal infections [2] [15].

In this work, we found that the presence of polybacterial communities, mainly constituted by commensals, is a quite diffuse characteristic of the ear canals of HA wearers. Thus, under particular situations, these polybacterial communities, either by exerting a synergistic effect on other microorganisms or by biofilm production, might concur to the development of acute otitis externa (AOE). Accordingly, it has been recently reported that pediatric, geriatric or immune-compromised patients are subjects at risk to develop AOE as well as systemic infections driven by microorganisms considered innocuous [21]. In this contest, it is worth to recall that biofilm production may constitute a scaffold for colonization of non-biofilm producing microorganisms, including pathogens, and thus this feature is considered an important virulence factor that allows microorganisms to better resist to the host immune response and to the antibiotic therapy [22].

This study is aimed at identifying and at comparing the microbial flora present in the ear canal of $57 \mathrm{HA}$ and of $33 \mathrm{nHA}$ users. Of these, 10 out of the $57 \mathrm{HA}$ and 12 out of the $33 \mathrm{nHA}$ users, presented signs of external auditory canal inflammation (mild/moderate). During microbial identification, we noticed significant differences in the distribution of bacterial species among the microbial flora of HA and nHA users (Table 1). Moreover, significant differences were also found for what it concerned the number of bacterial species isolated. A significantly higher number of microorganisms arose from swab samples of HA (about $10^{4}-10^{5} \mathrm{CFU} / \mathrm{ml}$ ) compared to nHA users (about $10^{2}-10^{3} \mathrm{CFU} / \mathrm{ml}$ ), indicating that carriage of HA devices per se may favors bacterial colonization of the ear canal. Although CoNS (Staphylococcus spp. in Table 1) strains are usually considered to be non-pathogenic, CoNSs were the commonest isolated organism (91.1\%) among HA or and of nHA users, presenting or not signs of inflammation. CoNS were isolated alone or in combination with other microorganisms (Table 1). Different forms of putative pathogenic bacteria (see Result section) were also isolated (Table 1). Interestingly, eight $S$. aureus and six $P$. aeruginosa strains were recovered only from $\mathrm{HA}$ and nHA users presenting signs 
of inflammation (respectively, 4/10 and 4/12; and 1/10 and 5/12). Although the number of subjects examined in this study is small, these results seem to indicate that inflammation may favor ear colonization by these pathogens that have been reported to be involved in the development of AOE [23]. Biofilm formation is considered an important virulence factor which allows bacteria to bind and to multiply within host tissues as well as to abiotic substrates (like the surfaces of HA devices) [24]. To ascertain whether bacterial species colonizing the ear canal of HA users were able to produce biofilm, we assayed representative bacterial species found on the ear canal of HA users with or without signs of inflammation. The results obtained indicated that biofilm production is a quite diffuse phenomenon among bacteria inhabiting the ear canal of HA users independently from inflammation. Interestingly, CoNS strains were also found to be strong biofilm producers. Since CoNS was the predominant microorganism recovered (see above) (Table 1), it is possible to envisage that the presence in the ear canal of these biofilm producers might influence the composition of the bacterial community in HA users and could provide optimal conditions for bacterial growth and antibiotic resistance.

Although the majority of isolates were components of the common normal flora of the ear, few putative pathogens were also recovered. Among these strains, $S$. aureus and $P$. aeruginosa were found to be strong biofilm producers.

Moreover, to further characterize $S$. aureus and $P$. aeruginosa strains, antibiotic-susceptibility and genetic relatedness (using an MLST scheme) were evaluated. For what it concerned antibiotic-resistance, the results obtained confirmed that the $P$. aeruginosa, compared to $S$. aureus presented a broader spectrum of antibiotic-resistance [25].

Nowadays the analysis of allelic variations of microorganisms is considered an important molecular tool (MLST test) used to precisely identify genetic relatedness among bacterial strains in order to study the insurgence and spread of specific microbial pathogens as well as of their epidemiology. A MLST assay was used to evaluate the genetic relatedness among isolates of $S$. aureus and of $P$. aeruginosa [17] [18]. PCR amplicons were generated and their sequences assigned to distinct alleles in order to determine sequence type (ST; see Materials and Methods for details). The six isolates of $P$. aeruginosa presented different ST values indicating that they are epidemiologically unrelated clones (Table 4), while $S$. aureus strains presented a lower level of genetic variability (Table 5). These results are in agreement with previous reports indicating high genetic variability for $P$. aeruginosa [18].

\section{Conclusion}

In conclusion, although the great majority of bacteria isolated in this study belonged to the common normal ear flora, some putative pathogens were also identified. For this reason, we would like to stress the need for HA users to carefully clean and disinfect their HA devices at least daily, in order to minimize the risk of developing serious ear canal infections. 


\section{Acknowledgements}

This work was supported by Ministero della Salute, Direzione Generale dei dispositivi medici e del servizio farmaceutico, CUP: B85J12000580001, CUP: J82I14001080001.

\section{Conflicts of Interest}

The authors declared no potential conflicts of interest with respect to the research, authorship, and publication of this article.

\section{References}

[1] Gates, G.A. and Mills, J.H. (2005) Presbycusis. The Lancet, 366, 1111-1120. https://doi.org/10.1016/S0140-6736(05)67423-5

[2] Karaca, C.T., Akçay, S.Ş., Toros, S.Z., Oysu, C., Verim, A., Çelebi, S. and Aksaray, S. (2013) External Auditory Canal Microbiology and Hearing Aid Use. American Journal of Otolaryngology, 34, 278-281. https://doi.org/10.1016/j.amjoto.2012.12.002

[3] Orji, F.T., Onyero, E. and Agbo, C.E. (2014) The Clinical Implications of Ear Canal Debris in Hearing Aid Users. Pakistan Journal of Medical Sciences, 30, 483-487. https://doi.org/10.12669/pjms.303.4742

[4] Rosenfeld, R.M., Schwartz, S.R., Cannon, C.R., Roland, P.S., Simon, G.R., Kumar, K.A., Huang. W.W., Haskell, H.W. and Robertson, P.J. (2014) Clinical Practice Guideline: Acute Otitis Externa Executive Summary. Otolaryngology-Head and Neck Surgery, 150, 161-168. https://doi.org/10.1177/0194599813517659

[5] Schaefer, P. and Baugh, R.F. (2012) Acute Otitis Externa: An Update. American Family Physician, 86, 1055-1061.

[6] Stroman, D.W., Roland, P.S., Dohar, J. and Burt, W. (2001) Microbiology of Normal External Auditory Canal. Laryngoscope, 111, 2054-2059. https://doi.org/10.1097/00005537-200111000-00035

[7] Frank, D.N., Spiegelman, G.B., Davis, W., Wagner, E., Lyons, E. and Pace, N.R. (2003) Culture-Independent Molecular Analysis of Microbial Constituents of the Healthy Human Outer. European Journal of Clinical Microbiology, 41, 295-303. https://doi.org/10.1128/JCM.41.1.295-303.2003

[8] Raza, S.A., Denholm, S.W. and Wong, J.C. (1995) An Audit of the Management of Acute Otitis Externa in an ENT Casualty Clinic. The Journal of Laryngology \& Otology, 109, 130-133. https://doi.org/10.1017/S0022215100129469

[9] Battikhi, M.N. and Ammar, S.I. (2004) Otitis Externa Infection in Jordan. Clinical and Microbiological Features. Saudi Medical Journal, 25, 1199-1203.

[10] Gerchman, Y., Patichov, R. and Zeltzer, T. (2012) Lipolytic, Proteolytic, and Cholesterol-Degrading Bacteria from the Human Cerumen. Current Microbiology, 64, 588-591. https://doi.org/10.1007/s00284-012-0113-8

[11] Kelly, K.E. and Mohs, D.C. (1996) The External Auditory Canal. Anatomy and Physiology. Otolaryngologic Clinics of North America, 29, 725-739.

[12] Walshe, P., Rowley, H. and Timon, C. (2001) A Worrying Development in the Microbiology of Otitis Externa. Clinical Otolaryngology and Allied Sciences, 26, 218-220. https://doi.org/10.1046/j.1365-2273.2001.00455.x

[13] Roland, P.S. (2002) Chronic Suppurative Otitis Media: A Clinical Overview. Ear, Nose \& Throat Journal, 81, 8-10. https://doi.org/10.1177/0194599813517659

[14] Geyer, M., Howell-Jones, R., Cunningham, R., et al. (2011) Health Protection Agency 
GP Microbiology Laboratory Use Group Consensus of Microbiology Reporting of Ear Swab Results to Primary Care Clinicians in Patients with Otitis Externa. British Journal of Biomedical Science, 68, 174-180. https://doi.org/10.1080/09674845.2011.11730346

[15] Ahmad, N., Etheridge, C., Farrington, M. and Baguley, D.M. (2007) Prospective Study of the Microbiological Flora of Hearing Aid Moulds and the Efficacy of Current Cleaning Techniques. The Journal of Laryngology \& Otology, 121, 110-113. https://doi.org/10.1017/S0022215106002222

[16] Eden, P.A., Schmidt, T.M., Blakemore, R.P. and Pace, N.R. (1991) Phylogenetic Analysis of Aquaspirillummagnetotacticum Using Polymerase Chain Reaction-Amplified 16SrRNA-Specific DNA. International Journal of Systematic Bacteriology, 41, 324-325. https://doi.org/10.1099/00207713-41-2-324

[17] Enright, M.C., Day, N.P., Davies, C.E., Peacock, S.J. and Spratt, B.G. (2000) Multilocus Sequence Typing for Characterization of Methicillin-Resistant and Methicillin-Susceptible Clones of Staphylococcus aureus. Journal of Clinical Microbiology, 38, 1008-1015.

[18] Curran, B., Jonas, D., Grundmann, H., Pitt, T. and Dowson, C.G. (2004) Development of a Multilocus Sequence Typing Scheme for the Opportunistic Pathogen Pseudomonas aeruginosa. Journal of Clinical Microbiology, 42, 5644-5649. https://doi.org/10.1128/JCM.42.12.5644-5649.2004

[19] Conte, M.P., Longhi, C., Marazzato, M., Conte, A.L., Aleandri, M., Lepanto, M.S., Zagaglia, C., Nicoletti, M., Aloi, M., Totino, V., Palamara, A.T. and Schippa, S. (2016) The Adherent/Invasive Escherichia coli Strain LF82 Invades and Persists in Human Prostate Cell Line RWPE-1, Activating a Strong Inflammatory Response. Infection and Immunity, 17, 3105-3113. https://doi.org/10.1186/1756-0500-7-748

[20] Stepanović, S., Vuković, D., Hola, V., Di Bonaventura, G., Djukić, S., Ćirković, I. and Ruzicka, F. (2007) Quantification of Biofilm in Microtiter Plates: Overview of Testing Conditions and Practical Recommendations for Assessment of Biofilm Production by Staphylococci. APMIS, 115, 891-899.

https://doi.org/10.1111/j.1600-0463.2007.apm_630.x

[21] McWilliams, C.J., Smith, C.H. and Goldman, R.D. (2012) Acute Otitis Externa in Children. Canadian Family Physician, 58, 1222-1224.

[22] Paluch-Oleś, J., Magryś, A., Kozioł-Montewka, M., Niedzielski, A., Niedźwiadek, J., Niedzielska, G. and Kotowski, M. (2011) The Phenotypic and Genetic Biofilm Formation Characteristics of Coagulase-Negative Staphylococci Isolates in Children with Otitis Media. International Journal of Pediatric Otorhinolaryngology, 75, 126-130. https://doi.org/10.1016/j.ijporl.2010.10.025

[23] Berni, E., Scott, L.A., Jenkins-Jones, S., Hanka De Voogd, H., Rocha, M.S., Butler, C.C., Morgan, C.L., Craig, J. and Currie, C.J. (2016) Non-Response to Antibiotic Treatment in Adolescents for Four Common Infections in UK Primary Care 1991-2012: A Retrospective, Longitudinal Study. Antibiotics, 5, 25. https://doi.org/10.3390/antibiotics5030025

[24] Torretta, S., Drago, L., Marchisio, P., Mattina, R., Clemente, I.A. and Pignataro, L. (2011) Diagnostic Accuracy of Nasopharyngeal Swabsin Detecting Biofilm-Producing Bacteria in Chronic Adenoiditis: A Preliminary Study. Otolaryngology-Head and Neck Surgery, 144, 784-788. https://doi.org/10.1177/0194599810394955

[25] Morita, Y., Tomida, J. and Kawamura, Y. (2014) Responses of Pseudomonas aeruginosa to Antimicrobials. Frontiers in Microbiology, 4, 422. https://doi.org/10.3389/fmicb.2013.00422 